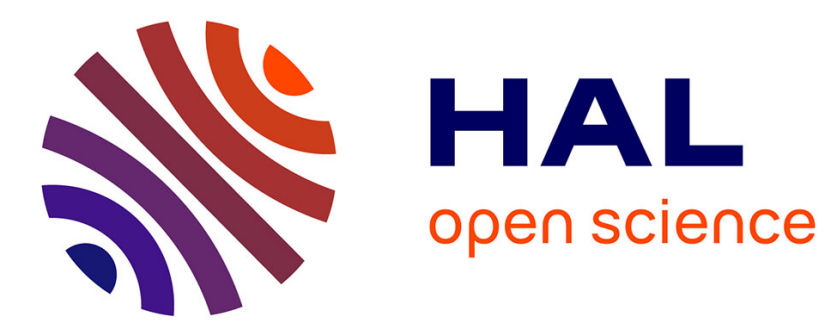

\title{
Quadratic Objective Functions for Dichromatic Model Parameters Estimation
}

\author{
Alexandre Krebs, Yannick Benezeth, Franck Marzani
}

\section{To cite this version:}

Alexandre Krebs, Yannick Benezeth, Franck Marzani. Quadratic Objective Functions for Dichromatic Model Parameters Estimation. International Conference on Digital Image Computing: Techniques and Applications, 2017, Sydney, Australia. hal-01606701

\section{HAL Id: hal-01606701 https://u-bourgogne.hal.science/hal-01606701}

Submitted on 2 Oct 2017

HAL is a multi-disciplinary open access archive for the deposit and dissemination of scientific research documents, whether they are published or not. The documents may come from teaching and research institutions in France or abroad, or from public or private research centers.
L'archive ouverte pluridisciplinaire HAL, est destinée au dépôt et à la diffusion de documents scientifiques de niveau recherche, publiés ou non, émanant des établissements d'enseignement et de recherche français ou étrangers, des laboratoires publics ou privés. 


\title{
Quadratic Objective Functions for Dichromatic Model Parameters Estimation
}

\author{
Alexandre Krebs, Yannick Benezeth and Franck Marzani \\ Le2i FRE2005, CNRS, Arts et Métiers \\ Univ. Bourgogne Franche-Comté \\ F-21000 Dijon, France \\ Email: name.lastname@u-bourgogne.fr
}

\begin{abstract}
In this paper, we present a novel method to estimate dichromatic model parameters from a single color image. Estimation of reflectance, shading and specularity has many applications such as shape recovery, specularity removal and facilitates classical image processing and computer vision tasks such as segmentation or classification. Our method is based on two successive and independent constrained quadratic programming steps to recover the parameters of the model. Compared to recent methods, our approach has the advantage to transform a complex inverse problem into two parralelizable optimization steps that are much easier to solve. We have compared our method with recent works in the field to assess its robustness and accuracy on accessible datasets. The advantages of our method are shown by analyzing qualitatively and quantitatively the resulting image decompositions.
\end{abstract}

\section{INTRODUCTION}

An image acquired by a sensor is the result of reflections of light on a surface. Reflection of light is either diffuse or specular. Diffuse reflection is due to subsurface scattering while specular reflection is a mirror-like reflection. Thus, a challenging task is to isolate the contributions of these phenomena. The resulting decomposition into intrinsic images gives useful information about the shape, the color of an object and the geometry of the scene. For example, the color of an object can be used for segmentation or classification and the shape and the geometry give 3D information about the environment from 2D images. The modelling of surface reflectance and the estimation of photometric invariants is a topic of significant importance and several reflectance models have been proposed. Because of its simplicity, the dichromatic model is widely used in the literature.

The dichromatic model expresses the idea that an image can be split into a diffuse and a specular part. The diffuse component is the result of light being reflected equally in all directions and the specular part is the light being exactly reflected in the viewing direction. The model is mathematically defined by the equation:

$$
I(u, \lambda)=l(\lambda)(g(u) S(u, \lambda)+k(u))
$$

where $I$ is the input image, $l$ is the spectrum of the illuminant multiplied by the sensor sensitivity, $g$ is the shading factor, $k$ is the specular coefficient and $S$ is the reflectance image. $u$ and $\lambda$ denote respectively the pixel location and the wavelength. In expression (1), the diffuse part is $l(\lambda) g(u) S(u, \lambda)$ and the specular part is $l(\lambda) k(u)$. At specular pixels (when $g$ is low compared to $k$ ) the surface acts like a mirror and the pixel color is directly proportional to $l$. Inversely, if $k$ is low, the pixel color is proportional to the multiplication of the color of the object $S$ and $l$.

Most of the time the spectrum of the illuminant can be known and (1) becomes:

$$
R(u, \lambda)=\frac{I(u, \lambda)}{l(\lambda)}=g(u) S(u, \lambda)+k(u) .
$$

This simplification shows that the reflectance spectra $S$ is known within a scalar and an offset. $l$ can be obtained thanks to one of the reference methods taken from literature like the White-Patch method, the Grey-World method or the GreyEdge method [1] [2]. New deep learning based algorithms compete these methods like the Convolutional Neural Network of Bianco et al. [3] or the mixed pooling neural networks of Fourure et al. [4].

The dichromatic model was first proposed by Shafer in 1985 for modeling dielectric objects [5]. This model is actually a special case of the Bidirectional Reflectance Distribution Function (BRDF) simplified with the assumption of a uniform illumination across the spatial domain. BRDF is the common basis of various phenomenological models like the lambertian model (Lambert 1760) or the Wolff model, used to explain reflections on smooth dielectric surfaces (Wolff 1994) [1]. Barron et al. have presented the Shape, Illumination and Reflectance From Shading (SIRFS) model in [6]. SIRFS is different from the previous list because it is parametrized with a rendering engine, which produces a log-shading image from a depth map, and a spherical-harmonic model of illumination.

Even if the dichromatic model is rather simple, its inversion is still complex: the inversion process is an underdetermined problem. One single image could have been obtained by a large combination of illumination, shapes and reflectances as explained by Barron et al. [6] and Adelson et al. [7]. As a consequence, it is common to increase the amount of data available, for example by combining multiple views of the scene. Using multiple images makes easier the separation of diffuse and specular components. For example, Umemaya et $a l$. use a rotating polarizer to acquire several images and then apply the Independent Component Analysis (ICA) on the images assuming the probabilistic independence between 
diffuse and specular components [8]. Feris et al. use multiflash images to reduce specularity [9].

Another way to overcome the underdetermination is to work on a single image. With a single RGB image, the methods are often based on neighborhood analysis: Tan et al. have described a specular-to-diffuse mechanism which is applied on local neighborhood having the same reflectance [10], [11]. Fast recovery of intrinsic images from a single image already exists with the methods of Yoon et al. [12] or Yang et al. [13]. Yoon et al. create specular-free 2-channel images and Yang et al. use guided filtering to remove specular [14].

As explained previously, solving the equation (2) is an underdetermined problem, there are more unknowns than equations. There is exactly $N_{p} \times N_{c}$ equations and $N_{p}+N_{p} \times N_{c}+N_{p}$ unknowns with $N_{p}$ as the number of pixels and $N_{c}$ as the number of channels. Other single image based approaches use constraints to overcome the underdetermination. Barron et al. have expressed priors on the illumination, the reflectance and the shape of an object [15] [6]. These constraints act as soft constraints and even if we are not using the same model, we use some of his ideas to build our own optimization algorithm. For example, Barron et al. explain that surfaces tend to be smooth, thus the image $g$ is also a smooth image. This idea is confirmed by RoblesKelly et al. [16] and Huynh et al. [17]. They use respectively the derivative of $g$ and the mean curvature to express the smoothness of $g$. Another interesting idea is that there is a small number of reflectances in an image, the palette used for an image tends to be small [6].

Recent work have expanded the recovery of intrinsic images to multispectral images. For example, Fu et al. [18] have applied Orthogonal Subspace Projection to remove specularities. Robles-Kelly and Huynh [1], [2], [17] have also worked on multispectral images. They have proposed to minimize objective functions based on the dichromatic model to recover intrinsic images and have used the decomposition for skin recognition, material clustering or specularity removal.

We have developed a method, based on the dichromatic model to recover the shading, the specular and the reflectance intrinsic images from a single color image. In this method, the image is processed in two independent steps that are two simple constrained quadratic programming.

The rest of the paper is organized as follow: the method used to recover the intrinsic images is developed in section II and the comparative results are given in section III.

\section{Details of The METhods}

$\mathrm{Gu}$ et al. have used quadratic objective functions because of their simplicity in [16]. They solve the decomposition problem in two successive steps: first, recovering $g$ ( $N_{p}$ unknowns), then recovering $k$ ( $N_{p}$ unknowns). We also solve the problem in two quadratic programming steps which are detailed in parts $\mathrm{B}$ and $\mathrm{C}$. The difference with $\mathrm{Gu}$ et al. is that our two steps are independent and thus, there is no error propagation and the steps can be parallelized.
The section is organized in three parts. First a spectral similarity measure is described in part A. This spectral similarity measure is essential for the understanding of the constrained quadratic programming steps described in parts B and C.

\section{A. Spectral similarity measure}

The spectral metric must be scaling invariant. The Spectral Angle Mapper (SAM) is scaling invariant and works well in our case. SAM is defined as the arccosine of the dot product between two spectrum $S_{1}$ and $S_{2}$ divided by their norms [19]:

$$
\operatorname{SAM}\left(S_{1}, S_{2}\right)=\arccos \left(\frac{\sum_{i=1}^{N_{c}} s_{1 i} s_{2 i}}{\sqrt{\sum_{i=1}^{N_{c}} s_{1 i}^{2}} \sqrt{\sum_{i=1}^{N_{c}} s_{2 i}^{2}}}\right)
$$

where $s_{1 i}$ and $s_{2 i}$ denote the i-th value of the vector $S_{1}$ or $S_{2}$ respectively.

SAM returns a value between 0 and $\frac{\pi}{2} ; 0$ meaning that the spectra are identical within a scaling factor. In practice, the similarity between two spectra $S_{1}$ and $S_{2}$ is estimated using $\zeta\left(S_{1}, S_{2}\right)=\exp \left(\frac{-S A M\left(S_{1}, S_{2}\right)^{2}}{r}\right)$ with $r$ as the bandwidth parameter. The negative exponential scales the values between 0 and 1 and reinforces the similarity when the two spectra are close.

\section{B. Recovering the shading factor}

The first step of our method is to recover the shading factor $g$. To begin with, the case where two pixels $u$ and $v$ belong to the same material is considered. They have the same reflectance $S$ i.e.

$$
\forall \lambda, S(u, \lambda)=S(v, \lambda)
$$

Interestingly, the ratio of the std of $R$ can be expressed simply with the ratio of the shading factor $g$ :

$$
\alpha_{u, v}=\frac{\operatorname{std}(R(u,:), \lambda)}{\operatorname{std}(R(v,:), \lambda)}=\frac{g(u) \operatorname{std}(S(u,:), \lambda)}{g(v) \operatorname{std}(S(v,:), \lambda)}=\frac{g(u)}{g(v)} .
$$

This equality suggests that it is possible to write the objective function as the weighted square of the difference of $g(u)$ and $g(v) \alpha_{u, v}$ :

$$
f_{1}(g)=\sum_{u} \sum_{v \in N(u)} \zeta_{u, v}\left(g_{u}-g_{v} \alpha_{u, v}\right)^{2}
$$

where $N(u)$ denotes the neighborhood of $u, \zeta_{u, v}$ is an abbreviation for $\zeta(R(u,:), R(v,:)), g_{u}$ and $g_{v}$ stand respectively for $g(u)$ and $\mathrm{g}(\mathrm{v})$.

This objective function can be improved by considering the case where the pixels $u$ and $v$ do not belong to the same material. As $g$ tends to be smooth, it is encouraged to minimize the square of the difference of $g(u)$ and $g(v)$

$$
f_{2}(g)=\sum_{u} \sum_{v \in N(u)}\left(1-\zeta_{u, v}\right)\left(g_{u}-g_{v}\right)^{2} .
$$

There is a difficult case when two pixels $u$ and $v$ are grey: the standard deviation is close or equal to zero and thus the ratio $\alpha_{u, v}$ is not calculable. Moreover, according to (2), a 
grey pixel is a pixel for which $R(u, \lambda)$ is constant for every wavelength and thus cannot be separated into a diffuse part and a specular part as it only depends on $u$ and not on $\lambda$. In practice, when the two pixels are grey, the ratio of the means is used instead of the ratio of the standard deviations:

$$
\beta_{u, v}=\frac{\operatorname{avg}(R(u,:), \lambda)}{\operatorname{avg}(R(v,:), \lambda)} .
$$

This affirmation actually corresponds to the assumption that there is no specularity on grey objects i.e. that $k=0$. This leads to the third part of the objective function:

$$
f_{3}(g)=\sum_{u} \sum_{v \in N(u)} \zeta_{u, v} \zeta_{u} \zeta_{v}\left(g_{u}-g_{v} \beta_{u, v}\right)^{2}
$$

with $\zeta_{u}=\zeta(R(v,:), \mathbf{1})$ and $\overline{\zeta_{u}}=1-\zeta(R(v,:), \mathbf{1})$. The symbol 1 is a vector of ones. $\zeta_{u}$ indicates whether a spectrum is nearly grey (value close to 1 ) or not (value close to 0 ).

The final objective function is written so that all previously defined cases are well encompassed: $f_{1}$ and $f_{2}$ are rewritten with an additionnal factor that removes grey pixels:

$$
\begin{gathered}
f_{1}(g)=\sum_{u} \sum_{v \in N(u)} \zeta_{u, v} \overline{\zeta_{u} \zeta_{v}}\left(g_{u}-g_{v} \alpha_{u, v}\right)^{2} \\
f_{2}(g)=\sum_{u} \sum_{v \in N(u)}\left(1-\zeta_{u, v} \zeta_{u} \zeta_{v}-\zeta_{u, v} \overline{\zeta_{u} \zeta_{v}}\right)\left(g_{u}-g_{v}\right)^{2} .
\end{gathered}
$$

Finally, the objective function is written as the sum of the three functions previously defined:

$$
f_{\text {shading }}(g)=f_{1}(g)+f_{2}(g)+f_{3}(g) .
$$

As this objective function is the sum of quadratic functions, $f_{\text {shading }}$ is also quadratic which means that the objective function can be written in matrix form as:

$$
f_{\text {shading }}(g)=\boldsymbol{g}^{t} \times \boldsymbol{H}_{\text {shading }} \times \boldsymbol{g}
$$

where $\boldsymbol{H}_{\text {shading }}$ is a square $N_{p} \times N_{p}$ matrix. Because $\boldsymbol{H}_{\text {shading }}$ is not symmetric, it is better to symmetrize this matrix using $\frac{\boldsymbol{H}_{\text {shading }}+\boldsymbol{H}_{\text {shading }}{ }^{t}}{2}$ instead of $\boldsymbol{H}_{\text {shading. An }}$ advantage of this formulation is that $\boldsymbol{H}_{\text {shading }}$ is a sparse matrix because we are only working on a square neighborhood around each pixel.

Note that without any constraints, a solution of (13) could be trivially the zero vector. Note also that, in the dichromatic model there could be any balancing factor between $\boldsymbol{g}$ and $S$ i.e. if $S^{*}$ and $g^{*}$ are solution of the inverse problem, then $a S^{*}$ and $\frac{g *}{a}$ are also solution of the problem for any scalar $a$. Thus, the sum of the elements of $\boldsymbol{g}$ can be fixed to a certain constant $c$ with the constraint: $\boldsymbol{g}^{t} \times \mathbf{1}=c$.

Moreover, if a mask is associated with the image to invalidate some pixels, the constraint becomes $\boldsymbol{g}^{t} \times \boldsymbol{m}=c$ and $\boldsymbol{g}^{t} \times \overline{\boldsymbol{m}}=0$ where $\boldsymbol{m}$ is a binary vector and $\overline{\boldsymbol{m}}$ is the binary inverse of $\boldsymbol{m} . g$ is finally obtained by solving:

$$
\left\{\begin{array}{l}
g^{*}=\underset{g}{\operatorname{argmin}} f_{\text {shading }}(g) \\
\text { subject to } \\
g^{* t} \times m=c \\
g^{* t} \times \bar{m}=0 \\
g^{*} \geq 0
\end{array}\right.
$$

\section{Recovering the specular factor}

The second step of our method is to recover the specular factor $k$. The method described below is independent from the previous calculus of $g$, and thus, the two optimizations are perfectly interchangeable or can be parallelized to speed up the algorithm.

To begin, let us express the ratio between $R$ and its standard deviation as $J$ :

$$
\begin{aligned}
J(u, \lambda) & =\frac{R(u, \lambda)}{\operatorname{std}(R(u,:), \lambda)} \\
& =\frac{g(u) S(u, \lambda)}{\operatorname{std}(R(u,:), \lambda)}+\frac{k(u)}{\operatorname{std}(R(u,:), \lambda)} \\
& =\frac{S(u, \lambda)}{\operatorname{std}(S(u,:), \lambda)}+\frac{k(u)}{\operatorname{std}(R(u,:), \lambda)} .
\end{aligned}
$$

We also define a new variable $h$ as:

$$
h(u)=\frac{k(u)}{\operatorname{std}(R(u,:), \lambda)}-\operatorname{avg}(J(u,:), \lambda)
$$

As written in (4), if two pixels $u$ and $v$ belong to the same material, they have the same reflectance and the difference $h(u)-h(v)$ is zero:

$$
\begin{aligned}
h(u)-h(v) & =\frac{k(u)}{\operatorname{std}(R(u,:), \lambda)}-\operatorname{avg}(J(u,:), \lambda) \\
& -\frac{k(v)}{\operatorname{std}(R(v,:), \lambda)}+\operatorname{avg}(J(v,:), \lambda) \\
& =\frac{\operatorname{avg}(S(v,:), \lambda)}{\operatorname{std}(S(v,:), \lambda)}-\frac{\operatorname{avg}(S(u,:), \lambda)}{\operatorname{std}(S(u,:), \lambda)} \\
& =0 .
\end{aligned}
$$

Thus our objective function can be written as the square of the difference between $h(u)$ and $h(v)$ weighted by $\zeta_{u, v}$ to express the similarity of the material and $\overline{\zeta_{u} \zeta_{v}}$ to express the fact that the pixels cannot be grey:

$$
f_{\text {specular }}(h)=\sum_{u} \sum_{v \in N(u)} \zeta_{u, v} \overline{\zeta_{u} \zeta_{v}}(h(u)-h(v))^{2} .
$$

This objective function is also quadratic and can be written as:

$$
f_{\text {specular }}(h)=\boldsymbol{h}^{t} \times \boldsymbol{H}_{\text {specular }} \times \boldsymbol{h}
$$

with $\boldsymbol{H}_{\text {specular }}$ a $N_{p} \times N_{p}$ symmetric matrix. 
Because of the physical constraint, $k$ is bounded below by 0 , all elements of $k$ must be positive. Moreover, the consideration of the minimum of $R$ over the wavelength

$$
\min (R(u, \lambda), \lambda)=g(u) \min (S(u, \lambda), \lambda)+k(u)
$$

shows that $k(u)$ is also upper-bounded by $\min (R(u, \lambda), \lambda)$.

Here, the physical positivity constraint bounds the values of $k(u)$ between 0 and $\min (R(u,:), \lambda)$. Thus, according to (18) $h(u)$ is also bounded:

$-\operatorname{avg}(J(u,:), \lambda) \leq h(u) \leq \frac{\min (R(u,:), \lambda)}{\operatorname{std}(R(u,:), \lambda)}-\operatorname{avg}(J(u,:), \lambda)$.

$h$ is obtained by solving the following system:

$$
\left\{\begin{array}{l}
h^{*}=\underset{h}{\operatorname{argmin}} f_{\text {specular }}(h) \\
\text { subject to } \\
-\operatorname{avg}(J, \lambda) \leq h^{*} \\
h^{*} \leq \frac{\min (R, \lambda)}{\operatorname{std}(R, \lambda)}-\operatorname{avg}(J, \lambda)
\end{array}\right.
$$

Once $g$ and $h$ are recovered, $k$ can be easily obtained with

$$
k(u)=(h(u)+\operatorname{avg}(J(u,:), \lambda)) \operatorname{std}(R(u,:), \lambda)
$$

and $S$ with

$$
S(u,:)=\frac{R(u,:)-k(u)}{g(u)} .
$$

To summarize, we have built two quadratic objective functions, to recover $g, k$ and indirectly $S$. The minimum of these functions can easily be found thanks to quadratic programming solvers.

\section{RESULTS AND DISCUSSION}

To assess the robustness of our method, we have compared it to current methods in the literature namely Barron et al. [6], Tan and Ikeuchi [11], Yang et al. [13], and two others developed by Robles-Kelly et al. [20] and [2]. In the next parts, we will use the following abbreviations to name each method:

- $L S$ for Least Square and corresponds to [20]

- KL for Robles-Kelly et al. [2]

- $T I$ for Tan and Ikeuchi et al. [11]

- Barron for Barron et al. method [6]

- Yang for Yang et al. method [13]

$L S$ employs shapelets to recover the shading of an image. $K L$ is based on objective functions with a regularization term that enforces the smoothness of $g$. TI creates specular-free images to separate reflection components of textured surfaces. Barron use priors to recover the most probable illumination, shape and reflectance and Yang uses guided image filtering to iteratively remove specularities.

We have tested the different algorithms on the Massachusetts Institue of Technology (MIT) intrinsic dataset [21], the same used by [6] and [16]. This dataset provides 20 images with a ground truth image for the reflectance $S$, the shading $g$ and the specular $k$. To interpret the results, we compare qualitatively the obtained images $g, S$ and $k$ with their respective ground truth $g_{\text {true }}, S_{\text {true }}$ and $k_{\text {true }}$ and results are also quantitatively compared.

\section{A. Qualitative analysis}

Table II shows six images from the dataset. For the sake of diversity, this subset is chosen to present images with smooth color changes (the apple and the pear) and with abrupt color changes (the sun, the phone, the panther and the raccoon). Similarly, the apple, the pear and the green part of the phone show clear specular spot while the sun, the panther and the raccoon do not.

$\log (k)$ images are shown in table III. It is easier to compare the logarithm of $k$ images because the logarithm increases the dynamic on low level pixels and thus reveals more details than $k$ images. The three columns show respectively the results for the apple, the pear and the phone. For the apple and the pear, $L S$ and our method seem to be the most efficient while TI overestimate the specular reflection, the image is saturated. For the phone, all methods are misled by the abrupt color changes. KL and Yang are also misled by the white part of the phone, which is not specularity. Note that the method based on the SIRFS model from Barron et al. is not presented as it does not provide a $k$ image.

Table IV presents shading images $g$ given by all methods. In this table, the advantages of our method appear: considering the apple and the pear, the specular spot still appear for $L S$, $K L$ and Yang while our method is more robust and is not corrupted by specularities. Considering the panther and the raccoon, our method makes successfully disappear the stars, ripples and other patterns while preserving the details of the shape like the hair of the raccoon. The same conclusion can be applied on the phone and the sun: strong gradients appear for methods $L S, K L$ and Yang that are not due to the shape of the object but are induced by color changes.

The smoothness term that was introduced in equation (7) have positive effects on our results and allows a real improvement compared to the recent works that have been done in the field.

\section{B. Quantitative analysis}

In addition to the qualitative analysis, the calculus of metrics between $g, S$ and $k$ is essential to prove the quality of the method.

As explained in part II.B there can be any scaling factor a between $g$ and $S$. Thus, we need to normalize $g$ and $g_{\text {true }}$ so that the sum of all pixels is 1 to be sure that they are scaled in the same way. After normalization, the Sum of Squared Errors (SSE) is computed to compare the two images.

For $S$ and $S_{\text {true }}$ the mean value of the SAM angles is computed. SAM is already a scaling invariant metric thus, there is no need to normalize $S$. Finally, for $k$ and $k_{t r u e}$, we compute the SSE without normalizing the images. Table I summarizes the results by giving the mean value over the 20 images of the MIT dataset. 
TABLE I

QUANTITATIVE RESULTS

\begin{tabular}{|c|c|c|c|c|c|c|}
\hline & LS & KL & TI & Barron & Yang & $\begin{array}{c}\text { Our } \\
\text { Method }\end{array}$ \\
\hline $\begin{array}{c}\text { SSE on } g \\
\text { normalized } \\
\left(\times 10^{-5}\right)\end{array}$ & 0.780 & 0.504 & 6.848 & 1.765 & 0.883 & $\mathbf{0 . 3 8 4}$ \\
\hline $\begin{array}{c}\text { SSE on } k \\
\left(\times 10^{13}\right)\end{array}$ & 0.208 & 2.282 & 2.391 & N/A & 0.357 & $\mathbf{0 . 0 1 7}$ \\
\hline SAM on $S$ & $\mathbf{0 . 0 2 1}$ & 0.041 & 1.177 & 0.032 & 0.025 & 0.030 \\
\hline
\end{tabular}

Our method outperforms the other methods for the calculus of $g$ and $k$ : concerning $g$ the proposed method has the lowest error with an SSE of $0.384 \times 10^{-5}$, with $K L\left(0.504 \times 10^{-5}\right)$ in second and $L S\left(0.780 \times 10^{-5}\right)$ in third position. This is consistent with the results shown previously in table IV.

Results on the SSE of $k$ are also favorable to our method with a SSE of $0.017 \times 10^{13}$ that is at least ten times lower than $L S\left(0.208 \times 10^{13}\right)$. In practice, this difference appears because $L S, K L, T I$ and Yang have significant errors on images with low specular level. This explains why, on average, the error is lower for our method.

Finally the results for $S$ show that our method is only ranked third with a mean angular error of 0.030 radians (1.7 degree). However, it should be noted that the mean angular error is quite low for all methods excepting for TI which probably suffers from bad cross-plateform adaptation.

\section{CONCLUSION}

In this paper, we have presented a novel method to recover the intrinsic parameters of the dichromatic model using a single color image. This method has better or similar accuracy to recent advances in the field, especially for the recovery of the shading factor $g$. Adding smoothness constraints helps to improve the quality of the shading recovery. The specific architecture of our algorithm, i.e. two simple constrained quadratic programming steps, open opportunities in the field to create memory and time efficient algorithms for the recovery of intrinsic images. The introduced method was also built with the idea to be directly transposable to multispectral or hyperspectral images. Therefore, the extension of the proposed method to this type of image forms part of our future work.

\section{ACKNOWLEDGMENT}

This study was supported by the French Research National Agency (ANR) program EMMIE under the grant agreement 15-CE17-0015.

\section{REFERENCES}

[1] A. Robles-Kelly and C. P. Huynh, Imaging spectroscopy for scene analysis. Springer Science \& Business Media, 2012.

[2] C. P. Huynh and A. Robles-Kelly, "A solution of the dichromatic model for multispectral photometric invariance," International Journal of Computer Vision, vol. 90, no. 1, pp. 1-27, 2010.

[3] S. Bianco, C. Cusano, and R. Schettini, "Color constancy using cnns," in Proceedings of the IEEE Conference on Computer Vision and Pattern Recognition Workshops, 2015, pp. 81-89.
[4] D. Fourure, R. Emonet, E. Fromont, D. Muselet, A. Trémeau, and C. Wolf, "Mixed pooling neural networks for color constancy," in Image Processing (ICIP), 2016 IEEE International Conference on. IEEE, 2016, pp. 3997-4001.

[5] S. A. Shafer, "Using color to separate reflection components," Color Research \& Application, vol. 10, no. 4, pp. 210-218, 1985.

[6] J. T. Barron and J. Malik, "Shape, illumination, and reflectance from shading," IEEE Transactions on Pattern Analysis and Machine Intelligence, vol. 37, no. 8, pp. 1670-1687, 2015.

[7] E. H. Adelson and A. P. Pentland, "The perception of shading and reflectance," Perception as Bayesian inference, pp. 409-423, 1996.

[8] S. Umeyama and G. Godin, "Separation of diffuse and specular components of surface reflection by use of polarization and statistical analysis of images," IEEE Transactions on Pattern Analysis and Machine Intelligence, vol. 26, no. 5, pp. 639-647, 2004.

[9] R. Feris, R. Raskar, K.-H. Tan, and M. Turk, "Specular reflection reduction with multi-flash imaging," in Computer Graphics and Image Processing, 2004. Proceedings. 17th Brazilian Symposium on. IEEE, 2004, pp. 316-321.

[10] R. T. Tan, K. Nishino, and K. Ikeuchi, "Separating reflection components based on chromaticity and noise analysis," IEEE Transactions on Pattern Analysis and Machine Intelligence, vol. 26, no. 10, pp. 1373-1379, 2004.

[11] R. T. Tan and K. Ikeuchi, "Separating reflection components of textured surfaces using a single image," IEEE Transactions on Pattern Analysis and Machine Intelligence, vol. 27, no. 2, pp. 178-193, 2005.

[12] K.-J. Yoon, Y. Choi, and I. S. Kweon, "Fast separation of reflection components using a specularity-invariant image representation," in International Conference on Image Processing. IEEE, 2006, pp. 973-976.

[13] Q. Yang, S. Wang, and N. Ahuja, "Real-time specular highlight removal using bilateral filtering," European Conference on Computer Vision, pp. 87-100, 2010.

[14] K. He, J. Sun, and X. Tang, "Guided image filtering," IEEE Transactions on Pattern Analysis and Machine Intelligence, vol. 35, no. 6, pp. 1397 1409, 2013.

[15] J. T. Barron and J. Malik, "Color constancy, intrinsic images, and shape estimation," in European Conference on Computer Vision. Springer, 2012, pp. 57-70.

[16] L. Gu and A. Robles-Kelly, "A quadratic optimisation approach for shading and specularity recovery from a single image," in International Conference on Image Processing (ICIP). IEEE, 2016, pp. 4072-4076.

[17] C. P. Huynh and A. Robles-Kelly, "Optimal solution of the dichromatic model for multispectral photometric invariance," in Joint IAPR International Workshops on Statistical Techniques in Pattern Recognition (SPR) and Structural and Syntactic Pattern Recognition (SSPR). Springer, 2008, pp. 382-391.

[18] Z. Fu, R. T. Tan, and T. Caelli, "Specular free spectral imaging using orthogonal subspace projection," in 18th International Conference on Pattern Recognition, vol. 1. IEEE, 2006, pp. 812-815.

[19] A. Galal, H. Hasan, and I. Imam, "Learnable hyperspectral measures," Egyptian Informatics Journal, vol. 13, no. 2, pp. 85-94, 2012.

[20] L. Gu, A. A. Robles-Kelly, and J. Zhou, "Efficient estimation of reflectance parameters from imaging spectroscopy," IEEE Transactions on Image Processing, vol. 22, no. 9, pp. 3648-3663, 2013.

[21] R. Grosse, M. K. Johnson, E. H. Adelson, and W. T. Freeman, "Ground truth dataset and baseline evaluations for intrinsic image algorithms," in 12th International Conference on Computer Vision. IEEE, 2009, pp. 2335-2342. 
TABLE II

EXAMPLES OF IMAGES EXTRACTED FROM THE MIT DATABASE
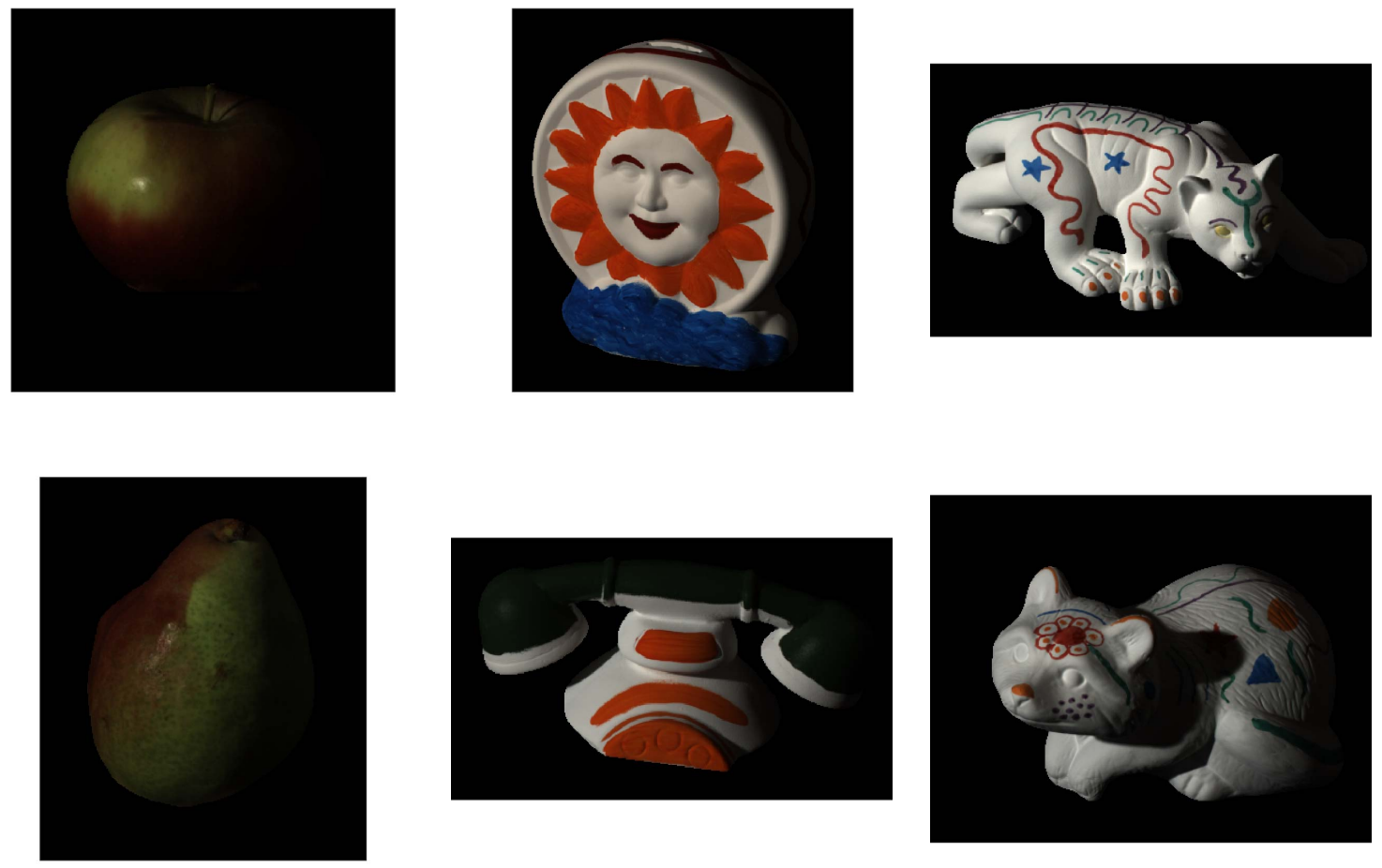
TABLE III

Qualitative Results For SPECUlar Reflection $k$

Apple
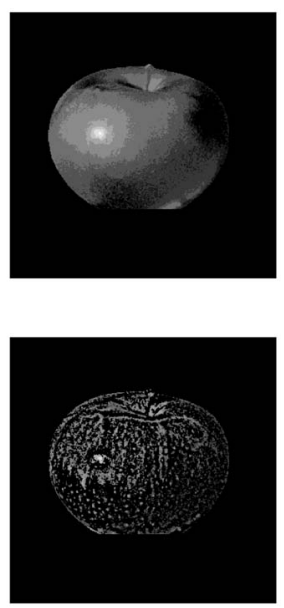

KL
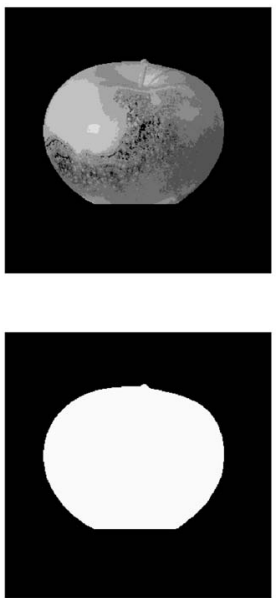

TI
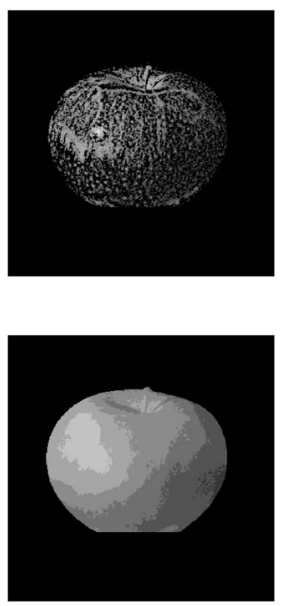

Pear
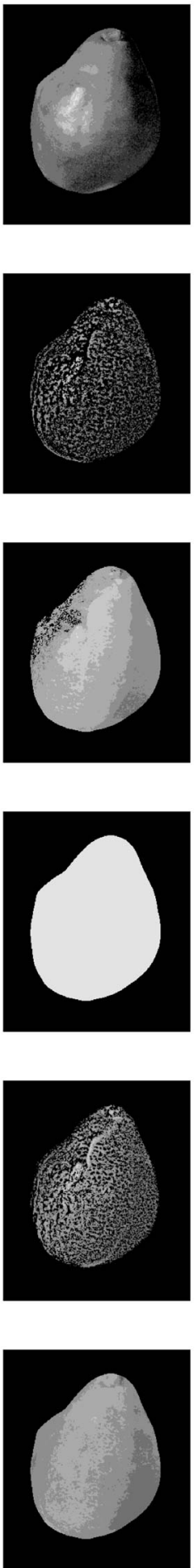
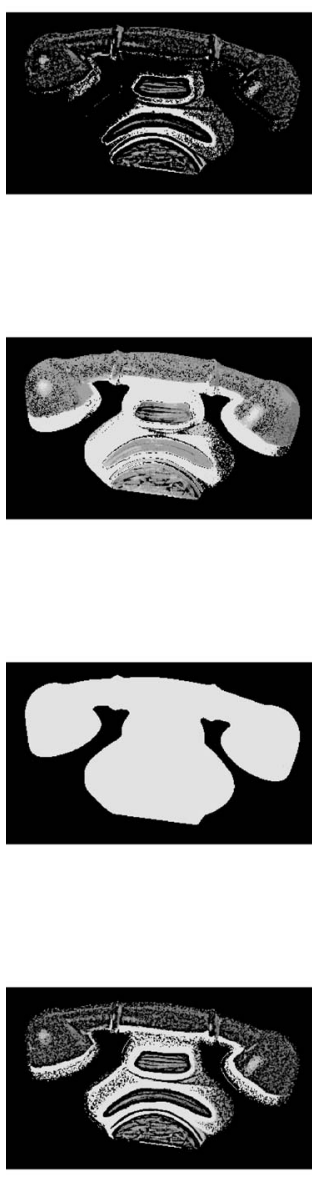

Phone
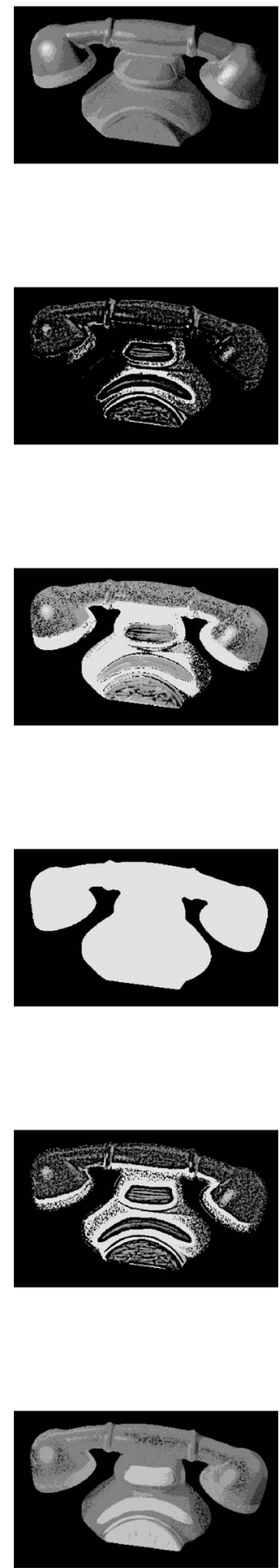
TABLE IV

QUALITATIVE RESULTS FOR THE SHADING FACTOR $g$

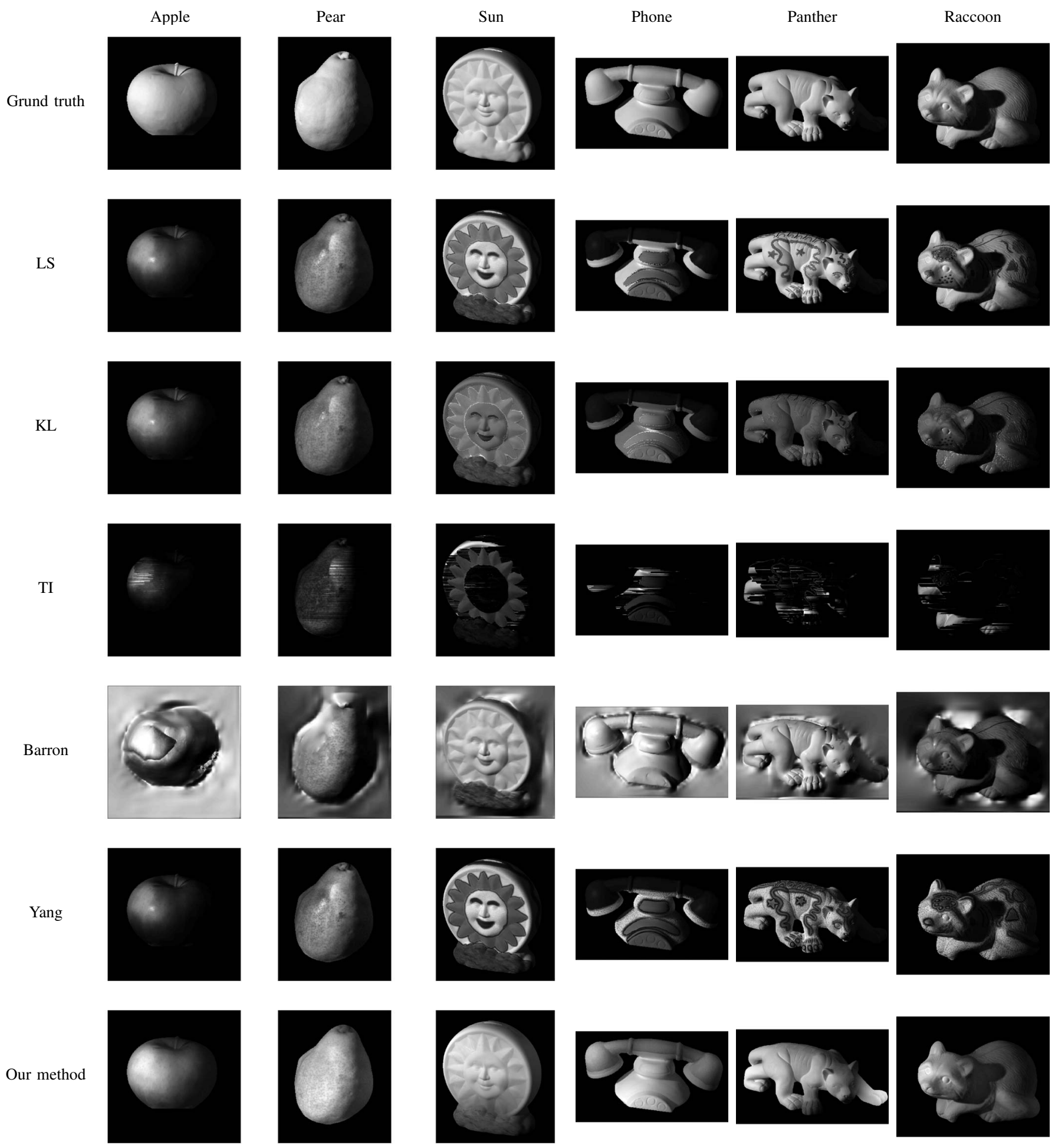

